\title{
Uso de fosfato para remoção de arsênio de solo contaminado
}

\author{
Waleska G. P. da Silva ${ }^{1}$, Nelson M. B. do Amaral Sobrinho ${ }^{2} \&$ Nelson Mazur ${ }^{2}$
}

\begin{abstract}
RESU MO
Quando da opção do procedimento de remediação de solos contaminados com metais pesados para determinado sítio, deve-se levar em consideração dados do sítio e do contaminante, o grau de contaminação, o uso futuro da área e a viabilidade econômica do processo de remediação. A Cia. Mercantil Ingá, localizada no município de Itaguaí, RJ, extraía zinco da calamina através do processo de hidrometalurgia. Durante 31 anos a empresa dispôs, no seu pátio e de forma inadequada, cerca de 3 milhões de toneladas de resíduo perigoso. U ma das etapas do processo de extração do zinco consistia na adição de trióxido de arsênio para a remoção de impurezas, tendo-se verificado contaminação do solo com arsênio de áreas próximas ao local de disposição do resíduo. Dentro deste contexto se avaliou, no presente estudo, a técnica de remediação ex situ, através da remoção por lavagem de solo contaminado com arsênio, e o tratamento do efluente gerado da lavagem. 0 fracionamento mostrou que a diminuição do pH aumentou a retenção do arsênio. 0 diidrogenofosfato de potássio $0,4 \mathrm{~mol}_{\mathrm{L}-1}^{-1}$ mostrou-se eficiente na remoção por dessorção do arsênio presente no solo contaminado, conseguindo dessorver cerca de 70\% deste elemento em pH 6,2. 0 cloreto férrico foi mais eficiente que 0 sulfato de alumínio para a remoção por coagulação, do arsênio do efluente da lavagem do solo.
\end{abstract}

Palavras-chave: remediação ex situ, Cia. M ercantil Ingá, coagulação, cloreto férrico, sulfato de alumínio

\section{Use of phosphate for removal of arsenic from contaminated soil}

\begin{abstract}
Several remediation procedures for soils contaminated with heavy metals are based on decontamination or stabilization. The selection of one of them for a given site should take data regarding the site and the contaminating agent, contamination degree, future use of the site and the economic viability of remediation process into account. The Mercantil Ingá Company, located in the municipality of Itaguaí - RJ, Brazil, has extracted zinc from calamine through hydrometallurgy. During 31 years, the company improperly disposed of about three million tons of hazardous residues in its area. 0 ne of the stages of the zinc extraction process consists of the addition of arsenic trioxide for the removal of impurities and the soil was contaminated with arsenic from areas near sites where the hazardous residues were disposed. In this context, this study evaluated the ex situ remediation technique through removal by leaching the contaminated soil with arsenic, and the treatment of the effluent generated. The fractioning showed that the $\mathrm{pH}$ reduction increased the arsenic retention. The potassium dihydrogenophosphate concentration in $0.4 \mathrm{~mol} \mathrm{~L}^{-1}$ seemed to be efficient in the removal of the arsenic present in the contaminated soil, achieving the dissolution of about $70 \%$ of this element at pH 6.2. The ferric chloride was more efficient than the aluminum sulphate for the removal, through coagulation, of the arsenic present in the effluent generated in the soil leaching.
\end{abstract}

Key words: remedition ex situ, Cia. Mercantil Ingá, coagulation, ferric chloride, aluminum sulphate 


\section{INTRODUÇão}

Diversos procedimentos de remediação dos metais pesados no solo são baseados na descontaminação (Santos et al., 2007) ou na estabilização (Yang, 2006).

$\mathrm{Na}$ escolha de um deles para determinado sítio deve-se levar em consideração, além da dimensão do impacto provocado pelo contaminante no ecossistema, dados do sítio e do contaminante, o grau de contaminação, o uso futuro da área e a viabilidade econômica do processo de remediação (Cunningham \& Lee, 1995). Todos os programas de remediação in situ seguem uma estratégia de contenção do contaminante (estabilização) (Mesquita et al., 2006) ou de redução do contaminante (limpeza) (Santos et al., 2006). Quando a contaminação é elevada e concentrada em área reduzida tem-se adotado estratégias ex situ (Kumpiene et al., 2006). Nesse tipo de estratégia o solo é removido e tratado em um sistema em separado (tanques de reação ou reatores, por exemplo); na própria área (on site), ou em uma instalação situada em outro local (off site) e, posteriormente, é feita a redisposição. As técnicas mais utilizadas, são: lavagem do solo e eletro-osmose, dentre outras (Cunningham \& Own, 1996). Para a remoção do contaminante através da lavagem do solo pode-se utilizar soluções com: sais neutros concentrados (Alam et al., 2007); soluções ácidas (Leist et al., 2000), e agentes que promovam a dessorção do contaminante (Fayiga \& Ma, 2006).

O arsênio é um elemento extremamente tóxico (Mahimairaja et al., 2005), cujas disponibilidade e toxicidade dependem da forma química em que se encontra no meio (Sadiq, 1997). Existe uma similaridade entre a química do arsênio e do fósforo, formando nos solos oxiânions (arseniato e fosfato) no estado de oxidação ${ }^{+5}$ (Quazi et al., 2005). As formas oxianiônicas de arsênio nos solos ricos em óxidos de ferro, estão envolvidas na adsorção específica desse elemento (Waychunas et al., 1993; Bhattacharya et al., 2007) e competem com os fosfatos (Wasay et al., 2000). A remoção do arsênio por dessorção com soluções de fosfato tem sido usada para lavagem deste elemento em solos contaminados (Codling \& Dao, 2007). Nesta técnica, é necessário que, posteriormente, se trate o efluente gerado sendo que a remoção do arsênio de efluentes contaminados tem sido realizada por coagulação, sedimentação e filtração (Nguyen et al., 2006). Os produtos $\mathrm{FeCl}_{3}$ (Tokunaga et al., 1997; Jang et al., 2007) e $\mathrm{Al}_{2}\left(\mathrm{SO}_{4}\right)_{3}$ (Silva, 2005) têm sido utilizados para remoção do arsênio por coagulação de efluentes líquidos.

A Cia. Mercantil Ingá é considerada a maior poluidora do Estado do Rio de Janeiro, visto que durante trinta e um anos de funcionamento dispôs, no pátio da empresa, cerca de três milhões de toneladas de resíduos sólidos, as quais representam grave ameaça ambiental a toda a região da Costa Verde, RJ. Este resíduo, além de apresentar altos teores de zinco, cádmio e chumbo (Santos et al., 2006), também contém arsênio como contaminante (Silva, 2005). Uma das etapas do processo de extração consistia na adição de trióxido de arsênio para remoção de impurezas; desta forma se tem verificado, também, a contaminação do solo com arsênio de áreas próximas ao local de disposição do resíduo perigoso da Ingá (Silva, 2005).
A otimização da extração do arsênio torna-se essencial e deverá preceder a utilização da técnica de lavagem do solo para a descontaminação.

Como objetivos este trabalho avaliou a dessorção do arsênio de solo de área próxima à disposição de resíduo perigoso, com fosfato e o tratamento do efluente gerado pela dessorção.

\section{MATERIAL E MÉTODOS}

Utilizou-se, para este trabalho, solo coletado no pátio de disposição de resíduo da Companhia Mercantil e Industrial Ingá, próximo ao antigo depósito de trióxido de arsênio (utilizado no processo de metalurgia com a finalidade de eliminar impurezas), em área localizada próxima ao porto de Itaguaí, no município de Itaguaí, RJ. A Companhia Mercantil e Industrial Ingá, situada no distrito de Ilha da Madeira, município de Itaguaí, RJ, era uma usina produtora de metal primário zinco e detentora de uma fábrica de tratamento de chapas metálicas, onde ocorria o processamento da mineração, redução e refino do metal A coleta do solo foi realizada em março de 2006 e consistiu em se retirar 50 amostras simples por hectare a uma profundidade de $0-40 \mathrm{~cm}$, imediatamente abaixo da pilha de resíduo, formando uma amostra composta. A pilha apresentava altura de $2 \mathrm{~m}$ e, para a realização da amostragem de solo, a pilha foi removida com auxílio de uma retroescavadeira. Em laboratório as amostras foram secadas ao ar, destorroadas e passadas por peneira de $2 \mathrm{~mm}$ e acondicionadas em sacos plásticos. A caracterização química e física do solo contaminado encontra-se na Tabela 1.

Tabela 1. Caracterização química e física do solo contaminado

\begin{tabular}{|c|c|c|c|c|c|c|c|c|}
\hline $\mathrm{Na}^{+*}$ & $\mathrm{Ca}^{+2^{*}}$ & $\mathrm{Mg}^{+2^{2}}$ & $H+A{ }^{* *}$ & $\overline{\mathbf{A l}^{+3^{*}}}$ & $\mathrm{pH} \mathrm{H} \mathrm{H}_{2} \mathrm{O}$ & C & Argila & $\mathbf{K}^{* * *}$ \\
\hline \multicolumn{5}{|c|}{$\mathrm{cmol}^{\mathrm{kg}^{-1}}$} & $1: 2,5$ & \multicolumn{2}{|c|}{$\mathrm{g} \mathrm{kg}^{-1}$} & $\mathrm{mg} \mathrm{kg}^{-1}$ \\
\hline 0,98 & 35,0 & 4,1 & 4,6 & 0,0 & 5,6 & 10,1 & 720 & 94 \\
\hline
\end{tabular}

Os teores pseudototais de $\mathrm{Cu}, \mathrm{Zn}, \mathrm{Mn}, \mathrm{Cd}, \mathrm{Pb}$ e Ni, foram determinados por digestão com água régia (EPA, 1986). O solo escavado também foi analisado como resíduo sólido, segundo Norma 10004 da ABNT (ABNT, 2004a) e os teores lixiviáveis NBR 10005 (ABNT, 2004b) avaliados pelo seguinte ensaio: NBR 10005 - Procedimento para obtenção de extrato lixiviado de resíduos sólidos (Tabela 2); esta Norma estima a capacidade de transferência de substâncias orgânicas e inorgânicas presentes no resíduo sólido, por dissolução em meio ácido (ácido acético), diferenciando os resíduos classificados pela ABNT NBR 10004 como classe I (perigosos) e classe II (não perigosos);

Determinaram-se, nos extratos obtidos na digestão e no teste de lixiviação os teores de $\mathrm{Cu}, \mathrm{Zn}, \mathrm{Mn}, \mathrm{Cd}, \mathrm{Pb}$ e Ni, por espectrofotometria de absorção atômica, empregando-se o equipamento VARIAN AA600 e o As por espectrofotometria de absorção atômica com geração de hidretos através de um acessório para geração de vapor modelo VGA 77 acoplado a um espectrofotômetro de absorção atômica. 
Tabela 2. Teores totais e lixiviáveis de $\mathrm{Zn}, \mathrm{Cd}, \mathrm{Mn}, \mathrm{Pb}, \mathrm{Fe}, \mathrm{Cu}, \mathrm{Ni}$ e As no solo do pátio do depósito da indústria Ingá $\left(\mathrm{mg} \mathrm{kg}^{-1}\right)$

\begin{tabular}{lcccccccc}
\hline \multicolumn{1}{c}{ Fração } & $\mathbf{Z n}$ & $\mathbf{C d}$ & $\mathbf{M n}$ & $\mathbf{P b}$ & $\mathbf{F e}$ & $\mathbf{C u}$ & $\mathbf{N i}$ & $\mathbf{A s}$ \\
Total & 22.295 & 175 & 5.526 & 2.487 & 279.987 & 123 & 32 & 6,27 \\
Valor de Intervenção(1) & 2.000 & 20 & - & 900 & - & - & - & 150 \\
Teste de lixiviação & - & 43,4 & - & 1,60 & - & - & - & 1,10 \\
Limite Máximo Teste de Lixiviação(2) $^{1}$ & - & 0,5 & - & 1,0 & - & - & - & 1,0 \\
\hline
\end{tabular}

(1) Valor de Intervenção para cenário industrial, segundo CETESB (2005); (2) Limite Máximo permitido no Teste de Lixiviação, segundo anexo G da Norma 10004 da ABNT (2004b)

As determinações de arsênio foram realizadas por espectrofotometria de absorção atômica por geração de hidretos, utilizando-se os seguintes parâmetros: comprimento de onda $=193,7 \mathrm{~nm} ;$ fenda $=0,2 \mathrm{~nm} ;$ corrente da lâmpada $=10 \mathrm{~mA}$; canal ácido $=\mathrm{HCl} 10 \mathrm{M}$; canal redutor $=\mathrm{NaBH}_{4} 0,6 \% \mathrm{~m} / \mathrm{v}$ $+\mathrm{NaOH} 0,5 \% \mathrm{~m} / \mathrm{v}+\mathrm{KI} 10 \% \mathrm{~m} / \mathrm{v}$, e LD $=0,02 \mu \mathrm{g} \mathrm{L} \mathrm{L}^{-1}$. O limite de detecção (LD) do método foi calculado multiplicando-se por três o desvio padrão do branco de todas as análises (15 repetições). Na curva de calibração obtida com a metodologia descrita acima, obteve-se um $\mathrm{R}^{2}$ de 0,9817 ; para a validação da metodologia analítica visando à determinação de arsênio, utilizou-se um material certificado de referência (NIST 1515, Apple Leaves); cinco repetições analíticas foram realizadas. A concentração de arsênio no material certificado foi de $0,038 \mathrm{mg} \mathrm{kg}^{-1}$ e o valor encontrado para a média das cinco repetições foi $0,040 \mathrm{mg} \mathrm{kg}^{-1}$ com desvio padrão de 0,005 .

Realizou-se um ensaio para verificar a influência do pH na distribuição geoquímica do arsênio presente no solo contaminado. Utilizaram-se potes plásticos contendo $500 \mathrm{~g}$ da amostra a qual foi umedecida a $80 \%$ da capacidade de campo e o pH ajustado com solução de $\mathrm{HCl} 0,05 \mathrm{~mol} \mathrm{~L}^{-1}$ ou $\mathrm{NaOH} 0,05 \mathrm{~mol} \mathrm{~L}^{-1}$, aos valores de: 4,3; 5,1; 5,6; 6,2 e 6,9, que permaneceram incubados durante 45 dias; após o período de incubação o material foi secado ao ar e novamente acondicionado em potes plásticos. $\mathrm{O}$ ensaio foi constituído de 5 tratamentos e 5 repetições totalizando 25 unidades experimentais; o delineamento utilizado foi o inteiramente casualizado; posteriormente, realizou-se o fracionamento utilizando-se a metodologia descrita por Wenzel et al. (2001) que consistiu de:

1) Extração com diidrogeno fosfato de amônio: agitaramse $2 \mathrm{~g}$ do material durante $16 \mathrm{~h}$, a $200{ }^{\circ} \mathrm{C}$, com $50 \mathrm{~mL}$ de diidrogeno fosfato de amônio $0,05 \mathrm{~mol} \mathrm{~L}^{-1}$, finda a agitação, filtrou-se o extrato em papel de filtro qualitativo;

2) Extração com tampão oxalato de amônio/ácido oxálico (pH 3,25); agitaram-se $2 \mathrm{~g}$ do material durante $4 \mathrm{~h}$, a $20{ }^{\circ} \mathrm{C}$, com $50 \mathrm{~mL}$ de tampão oxalato de amônio/ácido oxálico $0,2 \mathrm{~mol} \mathrm{~L}^{-1}$, no escuro, após a agitação, filtrou-se o extrato em papel de filtro qualitativo;

3) Extração com tampão oxalato de amônio/ácido oxálico ( $\mathrm{pH} 3,25)$ e ácido ascórbico. $2 \mathrm{~g}$ do material foram agitados por $30 \mathrm{~min}$, a $96{ }^{\circ} \mathrm{C}$, com $50 \mathrm{~mL}$ de tampão oxalato de amônio/ácido oxálico $0,2 \mathrm{~mol} \mathrm{~L}^{-1}$ e ácido ascórbico $0,1 \mathrm{~mol} \mathrm{~L}^{-1}$, no escuro, após a agitação, filtrou-se o extrato em papel de filtro qualitativo;

4) Digestão ácida: aqueceram-se, a $200{ }^{\circ} \mathrm{C}$, em becker de Teflon, em banho de areia, 0,5 g do material e $6 \mathrm{~mL}$ de ácido nítrico concentrado, até quase a secura (aproximadamente $30 \mathrm{~min}$ ). Esfriou-se o material e se lhe adicionaram $6 \mathrm{~mL}$ de ácido fluorídrico concentrado e $2 \mathrm{~mL}$ de ácido perclórico concentrado, aqueceu o a $200{ }^{\circ} \mathrm{C}$, até que não houvesse mais desprendimento de uma fumaça branca e o material estivesse quase seco. Após resfriamento do material adicionaramse $2 \mathrm{~mL}$ de ácido perclórico concentrado, aquecendo-o até quase a secura; foram adicionados, ainda, $25 \mathrm{~mL}$ de ácido clorídrico concentrado e aquecidos a $100{ }^{\circ} \mathrm{C}$, até que todo o resíduo se dissolvesse; filtrou-se o extrato em papel de filtro qualitativo e o volume foi completado para 50,00 mL.

Com os resultados obtidos através das extrações simples, estimaram-se as frações geoquímicas de arsênio no solo através das diferenças nas quantidades extraíveis, conforme apresentado a seguir:

F1 - Extraído com diidrogeno fosfato de amônio ==> Fração adsorvida não especificamente e especificamente.

F2 - (Extraído com tampão oxalato de amônio/ácido oxálico - Extraído com diidrogeno fosfato de amônio) ==> Fração ligada a oxi-hidróxidos de ferro e alumínio amorfos e pouco cristalinos.

F3 - (Extraído com tampão oxalato de amônio/ácido oxálico e ácido ascórbico - Extraído com tampão oxalato de amônio/ácido oxálico) ==> Fração ligada a oxi-hidróxidos de ferro e alumínio cristalinos.

F4 - (Extraído através de digestão ácida - Extraído com tampão oxalato de amônio/ácido oxálico e ácido ascórbico) ==> Fração associada a oclusão aos oxi hidróxidos de ferro e alumínio cristalinos e residual

Em outro ensaio se avaliou, nas mesmas amostras do solo contaminado com pH ajustado aos valores de: 4,3, 5,1, 5,6, 6,2, e 6,9, a dessorção do arsênio utilizando-se solução de fosfato $\left(\mathrm{KH}_{2} \mathrm{PO}_{4}\right)$ em diferentes concentrações $(0,0,0,1,0,2$, 0,3, 0,4, 0,6 e 0,8 $\mathrm{mol} \mathrm{L}^{-1}$ ) Escolheram-se esses valores de concentração com base no trabalho realizado por Wasay et al. (2000). O mesmo procedimento foi realizado usando-se água no lugar da solução de fosfato (controle/dose 0,0 ) nos seis valores de $\mathrm{pH}$. O ensaio foi constituído de 35 tratamentos e 5 repetições, totalizando 175 unidades experimentais; o delineamento utilizado foi o inteiramente casualizado.

No terceiro ensaio se avaliou a remoção do arsênio do efluente da dessorção, pelo fosfato $0,4 \mathrm{~mol} \mathrm{~L}^{-1} \mathrm{em} \mathrm{pH} 6,2$, do solo contaminado, através da "coagulação" com $\mathrm{FeCl}_{3}$ e com $\mathrm{Al}_{2}\left(\mathrm{SO}_{4}\right)_{3}$ em valores de $\mathrm{pH}$ de: 4,0, 5,0, 6,0, 7,0, 8,0, e 9,0. As doses utilizadas de $\mathrm{FeCl}_{3}$, foram de: 8,$5 ; 17 ; 25,5 ; 34 \mathrm{e}$ $42,5 \mathrm{mg} \mathrm{L}^{-1}$, e as doses de $\mathrm{Al}_{2}\left(\mathrm{SO}_{4}\right)_{3}$ foram de $70,140,210$, 280 e $350 \mathrm{mg} \mathrm{L}^{-1}$. Essas doses foram escolhidas com base no trabalho realizado por Hering et al. (1996). O ensaio foi realizado em beckers de $500 \mathrm{~mL}$ empregando-se um volume de 200 mL de solução; fez-se o ajuste do pH antes da adição do coagulante; após o ajuste do pH a coagulação se deu em três etapas: 1 min em agitação rápida (100 rpm); $30 \mathrm{~min}$ em 
agitação lenta (45 rpm) e 15 min em repouso cujas soluções foram filtradas em membranas de nitrato celulose $0,45 \mathrm{~mm}$, acidificadas com $\mathrm{HCl} 3 \mathrm{~mol} \mathrm{~L}^{-1}$ até $\mathrm{pH} 1$ e acondicionadas em frascos plásticos de polietileno; assim, este ensaio teve 60 tratamentos e 5 repetições, totalizando 300 unidades experimentais; o delineamento experimental utilizado foi o inteiramente casualizado.

\section{RESULTADOS E DISCUSSÃO}

Os teores de $\mathrm{Zn}$, $\mathrm{Cd}$ e $\mathrm{Pb}$, estiveram acima do Limite de Intervenção considerando-se um cenário industrial, segundo CETESB (2005) (Tabela 2). Os resultados do teste de lixiviação ABNT (2004a) permitiram classificar o solo escavado como resíduo Classe I - Perigoso, em função dos teores de $\mathrm{Cd}, \mathrm{Pb}$ e As estarem além dos padrões permitidos neste teste.

O fracionamento do arsênio nas diferentes formas químicas do solo é apresentado na Tabela 3. Na medida em que o pH decresceu, aumentou a concentração de arsênio associado às frações F3 e F4 mas, nas frações F1 e F2, o decréscimo do $\mathrm{pH}$ reduziu a quantidade extraída, resultados decorrentes, provavelmente, da maior adsorção deste elemento em valores mais baixos de $\mathrm{pH}$ (Mahimairaja et al., 2005). A redução do $\mathrm{pH}$ levou à migração de parte do arsênio das frações mais lábeis (F1 e F2) para as frações F3 e F4 reduzindo, como consequência, a solubilidade desse elemento e sua toxicidade. De acordo com Sadiq (1997), em ambientes naturais oxidados o arsênio se encontra, predominantemente, na forma de oxiânions; em valores de $\mathrm{pH}$ entre 4,3 e 7,0, a espécie predominante é a $\mathrm{H}_{2} \mathrm{AsO}_{4}$. O solo utilizado neste trabalho apresenta alto teor de ferro (Tabela 2), e na sua fração argila, óxidos, oxihidróxidos e hidróxidos de ferro, principalmente goethita (Pinheiro, 2003; Santos et al., 2007); esses minerais secundários apresentam valores de ponto de carga zero relativamente elevados. Provavelmente, a redução do $\mathrm{pH}$ a valores entre 4,3 e 5,6 conferiu uma carga líquida positiva à superfície e, desta forma, com a diminuição do $\mathrm{pH}$ natural do solo $(5,6)$ ocorreu uma afinidade maior desses oxiânions com a superfície dos óxidos, oxi hidróxidos e hidróxidos de ferro aumentando, consequentemente, a retenção e diminuindo a quantidade extraída. A dependência das cargas positivas nas superfícies das argilas com o $\mathrm{pH}$ pode ser a responsável pela associação encontrada entre o arsênio e os óxidos de ferro e de alumínio e as argilas silicatadas do tipo 1:1 (caulinita) (Mahimairaja et al., 2005). Em solos ácidos nos quais a superfície das partículas pode estar carregada positivamente, a adsorção específica de arsênio tem sido observada (Waychunas et al., 1993; Yang et al., 2007). O aumento da adsorção do arsênio com o decréscimo do pH e a associação do arsênio com os óxidos, oxi-hidróxidos e hidróxidos de ferro, tem sido observado em vários trabalhos (Spuller et al., 2007; Fayiga et al., 2007). Goldberg \& Glaubig (1988), reportaram que a adsorção dos oxiânions de arsênio ocorre por quimiossorção ou troca de ligantes nas superfícies das
Tabela 3. Teor de As $\left(\mu \mathrm{g} \mathrm{kg}^{-1}\right)$ nas diferentes frações geoquímicas em função do pH do solo contaminado

\begin{tabular}{crrrc}
\hline \multirow{2}{*}{$\mathbf{H}$} & \multicolumn{4}{c}{ Frações geoquímicas } \\
\cline { 2 - 5 } & $\mathbf{F 1}$ & $\mathbf{F 2}$ & $\mathbf{F 3}$ & $\mathbf{F 4}$ \\
4,2 & $311 \mathrm{dD}$ & $519 \mathrm{dC}$ & $1030 \mathrm{aB}$ & $4418 \mathrm{aA}$ \\
5,1 & $620 \mathrm{CC}$ & $887 \mathrm{cB}$ & $1030 \mathrm{aB}$ & $3741 \mathrm{bA}$ \\
5,6 & $891 \mathrm{bC}$ & $1129 \mathrm{bB}$ & $1147 \mathrm{aB}$ & $3111 \mathrm{cA}$ \\
6,2 & $882 \mathrm{bC}$ & $1562 \mathrm{aB}$ & $757 \mathrm{bC}$ & $3077 \mathrm{cA}$ \\
6,9 & $1018 \mathrm{aB}$ & $1500 \mathrm{aB}$ & $716 \mathrm{bC}$ & $3044 \mathrm{cA}$ \\
\hline
\end{tabular}

Letras minúsculas iguais nas colunas não diferem significativamente pelo Teste de Tukey a nível de $5 \%$ de probabilidade

Letras maiúsculas iguais nas linhas não diferem significativamente pelo Teste de Tukey a nível de $5 \%$ de probabilidade

argilas, caracterizando uma adsorção específica, conforme observado para fosfatos (Yang, 2006).

A dessorção de arsênio com fosfato do solo contaminado se acha na Tabela 4; verifica-se, na faixa de $\mathrm{pH}$ entre 4,3 e 5,6 , que em todas as concentrações de diidrogenofosfato de potássio, existe a mesma tendência, isto é, a quantidade de arsênio dessorvida não apresentou incremento significativo; entretanto, a partir de $\mathrm{pH}$ 5,6 este incremento foi significativo atingindo, na concentração de $0,4 \mathrm{~mol} \mathrm{~L}^{-1}$ e no pH 6,2, a máxima dessorção, correspondendo a $70 \%$ do total de arsênio no solo; em pH 7,0, ocorreu um decréscimo significativo da quantidade de arsênio removida na concentração de 0,4 mol L-1 de fosfato.

Tabela 4. Dessorção do arsênio $\left(\mathrm{mg} \mathrm{kg}^{-1}\right)$ do solo contaminado com soluções de $\mathrm{KH}_{2} \mathrm{PO}_{4}$ em diferentes valores de $\mathrm{pH}$

\begin{tabular}{cccccccc}
\hline \multirow{2}{*}{$\mathbf{p H}$} & \multicolumn{7}{c}{ Concentração de $\left.\mathbf{K H}_{\mathbf{2}} \mathbf{P O}_{\mathbf{4}} \mathbf{( m o l ~ L}^{-1}\right)$} \\
\cline { 2 - 8 } & $\mathbf{0 , 0}$ & $\mathbf{0 , 1}$ & $\mathbf{0 , 2}$ & $\mathbf{0 , 3}$ & $\mathbf{0 , 4}$ & $\mathbf{0 , 6}$ & $\mathbf{0 , 8}$ \\
4,3 & $89 \mathrm{aD}$ & $1162 \mathrm{bC}$ & $1332 \mathrm{bB}$ & $1634 \mathrm{cB}$ & $2338 \mathrm{bA}$ & $1100 \mathrm{bC}$ & $1080 \mathrm{bC}$ \\
5,2 & $77 \mathrm{aD}$ & $1189 \mathrm{bC}$ & $1487 \mathrm{bB}$ & $1872 \mathrm{cA}$ & $1914 \mathrm{CA}$ & $1235 \mathrm{bC}$ & $1205 \mathrm{bC}$ \\
5,6 & $50 \mathrm{aD}$ & $1282 \mathrm{bC}$ & $1650 \mathrm{bCB}$ & $2021 \mathrm{bCB}$ & $2577 \mathrm{bA}$ & $1250 \mathrm{bC}$ & $1150 \mathrm{bC}$ \\
6,2 & $41 \mathrm{aD}$ & $2739 \mathrm{aC}$ & $3751 \mathrm{aB}$ & $3598 \mathrm{aB}$ & $4084 \mathrm{aA}$ & $2424 \mathrm{aC}$ & $2370 \mathrm{aC}$ \\
7,0 & $52 \mathrm{aE}$ & $1275 \mathrm{bD}$ & $3618 \mathrm{aA}$ & $2776 \mathrm{bB}$ & $2880 \mathrm{bB}$ & $2220 \mathrm{aC}$ & $2120 \mathrm{aC}$ \\
\hline
\end{tabular}

Letras minúsculas iguais nas colunas não diferem significativamente pelo Teste de Tukey a nível de $5 \%$ de probabilidade

Letras maiúsculas iguais nas linhas não diferem significativamente pelo Teste de Tukey a nível de $5 \%$ de probabilidade

Em razão da sua alta afinidade com o oxigênio, o arsênio é comumente encontrado na forma de oxiânions na solução de solos oxidados (Sadiq, 1997). Segundo este autor, na faixa de pH entre 4,0 e 6,4, a espécie aniônica predominante é a espécie monovalente; em pH 6,9, que é o valor do $\mathrm{pKa}_{2}$ do ácido arsênico, as concentrações das espécies aniônicas monovalentes e divalentes são iguais; desta forma, pode-se inferir que a redução brusca da dessorção do arsênio a partir dos valores de $\mathrm{pH}$ acima do $\mathrm{pKa}_{2}$ pode estar associada ao aumento da concentração da espécie aniônica divalente, de menor solubilidade do que a espécie aniônica monovalente, formando compostos de menor solubilidade com os cátions presentes na solução solo (Masscheleyn et al., 1991). Não se pode descartar a possibilidade da solução de fosfato ter a sua solubilidade reduzida em valores de $\mathrm{pH}$ superiores a 6,0, ao formar compostos de baixa solubilidade, principalmente com o cálcio, que apresenta concentração 
muito elevada nesse solo contaminado (Silva, 2005) e, assim, reduzir sua capacidade extratora. Observa-se também que a dessorção aumentou até a concentração de $0,4 \mathrm{~mol} \mathrm{~L}^{-1}$ de diidrogenofosfato de potássio em todas as faixas de $\mathrm{pH}$, decrescendo significativamente a partir da concentração de $0,6 \mathrm{~mol} \mathrm{~L}^{-1}$, sendo que a quantidade dessorvida com soluções com concentrações superiores a $0,6 \mathrm{~mol} \mathrm{~L}^{-1}$ foi cerca de $50 \%$ inferior.

Os resultados da remoção do arsênio da solução após dessorção com $\mathrm{KH}_{2} \mathrm{PO}_{4}$, com $\mathrm{FeCl}_{3}$ e com $\mathrm{Al}_{2}\left(\mathrm{SO}_{4}\right)_{3}$ são apresentados nas Tabela 5.

Tabela 5. Porcentagem de arsênio no efluente da extração, removido em função do pH e da dose de $\mathrm{FeCl}_{3}$ e $\mathrm{Al}_{2}\left(\mathrm{SO}_{4}\right)_{3}$

\begin{tabular}{ccccccr}
\hline \multicolumn{7}{c}{$\mathbf{p H}$} \\
\cline { 2 - 8 } & $\mathbf{4 , 0}$ & $\mathbf{5 , 0}$ & $\mathbf{6 , 0}$ & $\mathbf{7 , 0}$ & $\mathbf{8 , 0}$ & $\mathbf{9 , 0}$ \\
8,5 & $85,7 \mathrm{aA}$ & $87,8 \mathrm{aA}$ & $86,1 \mathrm{aA}$ & $79,3 \mathrm{aB}$ & $73,3 \mathrm{aB}$ & $46,5 \mathrm{aC}$ \\
17,0 & $76,6 \mathrm{aA}$ & $79,8 \mathrm{bA}$ & $69,7 \mathrm{CA}$ & $79,0 \mathrm{aA}$ & $73,9 \mathrm{aA}$ & $47,2 \mathrm{aB}$ \\
25,5 & $82,1 \mathrm{aA}$ & $80,1 \mathrm{bA}$ & $76,9 \mathrm{cbB}$ & $74,6 \mathrm{aB}$ & $61,7 \mathrm{aC}$ & $52,1 \mathrm{aD}$ \\
34,0 & $81,6 \mathrm{aA}$ & $86,0 \mathrm{aA}$ & $81,5 \mathrm{bA}$ & $73,6 \mathrm{aB}$ & $70,1 \mathrm{aB}$ & $51,1 \mathrm{aC}$ \\
42,5 & $85,1 \mathrm{aA}$ & $80,7 \mathrm{bA}$ & $75,8 \mathrm{cbB}$ & $81,1 \mathrm{aA}$ & $61,0 \mathrm{aC}$ & $49,7 \mathrm{aD}$ \\
\hline & & \multicolumn{5}{c}{$\mathrm{Al}_{2}\left(\mathrm{SO}_{4}\right)_{3}\left(\mathrm{mg} \mathrm{L}^{-1}\right)(\%)$} \\
70 & $74,2 \mathrm{aA}$ & $81,9 \mathrm{aA}$ & $74,9 \mathrm{aA}$ & $62,8 \mathrm{aB}$ & $52,0 \mathrm{aB}$ & $3,6 \mathrm{bC}$ \\
140 & $57,9 \mathrm{bA}$ & $63,6 \mathrm{bA}$ & $55,5 \mathrm{aA}$ & $62,2 \mathrm{aA}$ & $53,0 \mathrm{aA}$ & $5,0 \mathrm{bB}$ \\
210 & $67,7 \mathrm{abA}$ & $64,1 \mathrm{bA}$ & $58,5 \mathrm{aB}$ & $54,2 \mathrm{aB}$ & $51,0 \mathrm{aB}$ & $13,8 \mathrm{aC}$ \\
280 & $66,8 \mathrm{abA}$ & $74,9 \mathrm{abA}$ & $66,7 \mathrm{aA}$ & $52,6 \mathrm{aB}$ & $46,1 \mathrm{aB}$ & $12,0 \mathrm{aC}$ \\
350 & $73,3 \mathrm{aA}$ & $65,3 \mathrm{aA}$ & $66,4 \mathrm{aA}$ & $65,9 \mathrm{aA}$ & $47,8 \mathrm{aB}$ & $9,5 \mathrm{aC}$ \\
\hline
\end{tabular}

Letras minúsculas iguais nas colunas não diferem significativamente pelo Teste de Tukey ao nível de $5 \%$ de probabilidade

Letras maiúsculas iguais nas linhas não diferem significativamente pelo Teste de Tukey ao nível de $5 \%$ de probabilidade

Verifica-se redução na remoção do arsênio com $\mathrm{FeCl}_{3}$ com o aumento do $\mathrm{pH}$, com reduções significativas a partir de pH 7,0; a menor remoção foi observada em pH 9, correspondendo aproximadamente a $50 \%$ do arsênio removido na faixa de pH entre 4 e 6; esta baixa remoção se deveu, sem dúvida, ao valor do ponto de carga zero dos óxidos de ferro. Segundo Sadiq (1997), o ponto de carga zero dos óxidos de ferro está em torno de $\mathrm{pH}$ 9,0. Como já discutido, em valores de $\mathrm{pH}$ acima do ponto de carga zero a superfície apresenta uma carga líquida negativa; logo, a adsorção do arsênio é reduzida e, consequentemente, a remoção por coagulação é menor; nota-se, também, que o aumento da dose do coagulante não aumentou de forma significativa a taxa de remoção do arsênio na faixa de pH estudada conforme observaram Hering et al. (1996).

A remoção do arsênio da solução com sulfato de alumínio foi menos efetiva que com o cloreto férrico (Tabela 5); a máxima remoção foi alcançada em valores de $\mathrm{pH}$ também entre 4,0 e 6,0, correspondendo a 74\% do arsênio total presente na solução e a menor remoção ocorreu também em pH 9,0 ; para a remoção com o sulfato de alumínio não se observou efeito da dose do coagulante.

A maior eficiência de remoção do arsênio com o cloreto férrico quando comparada com a pirita, tem sido verificada em vários trabalhos (Cheng et al., 1994; Bhattacharya et al., 2007; Jang et al., 2007).

\section{CONCLUSÕES}

1. O fracionamento geoquímico mostrou que a maior parte do arsênio presente no solo contaminado estava associada à fração associada à oclusão aos oxi hidróxidos de ferro e alumínio cristalinos e residual (F4).

2. Em valores mais elevados de pH, o arsênio se associou às frações químicas mais lábeis (fração adsorvida não especificamente e especificamente, F1 e fração ligada a oxi hidróxidos de ferro e alumínio amorfos e pouco cristalinos, F2), o que acarretou aumento da sua solubilidade e da toxicidade.

3. A diminuição do pH tornou o arsênio menos disponível, ou seja, mais retido nas superfícies dos óxidos, oxi-hidróxidos e hidróxidos de ferro, presentes no solo.

4. O diidrogenofosfato de potássio $0,4 \mathrm{~mol} \mathrm{~L}^{-1}$ mostrou-se eficiente na remoção por dessorção, do arsênio presente em solos contaminados, conseguindo dessorver cerca de $70 \%$ deste elemento em $\mathrm{pH} 6,2$.

5. O cloreto férrico foi mais eficiente que o sulfato de alumínio para a remoção, por coagulação, do arsênio do efluente da lavagem do solo.

\section{LITERATURA CITADA}

ABNT - Associação Brasileira de Normas Técnicas. Norma 10004 Resíduos sólidos - Classificação. São Paulo: ABNT, 2004a. 61p.

ABNT - Associação Brasileira de Normas Técnicas. Norma 10005., Procedimento para obtenção de extrato lixiviado de resíduos sólidos, São Paulo: ABNT, 2004b. 10p.

Alam, M.G.M.; Tokunaga, S.; Stagnitti, F. Removal of arsenic from contaminated soils using different salt extractants. Journal of Environmental Science and Health, Part A. v.42, n.4, p.447-451, 2007.

Bhattacharya P.; Mukherjee A.B.B.; Bundschuh J.; Zevenhoven R.; Loeppert, R.H. (ed.). Arsenic in soil and groundwater environment, v.9: Biogeochemical Interactions, Health Effects and Remediation (Trace Metals and other Contaminants in the Environment) 684p. 2007.

CETESB - Companhia de Tecnologia de Saneamento Ambiental. Valores orientadores para solos e águas subterrâneas no Estado de São Paulo. São Paulo: CETESB, 2005.4p

Cheng, R.; Liang, S.; Wang,H.; Beuhler, M. Enhanced coagulation for arsenic removal. Journal of American Water Works, v.86, n.9, p.79-90, 1994.

Codling, E. E.; Dao, T. H. Short Term Effect of Lime, Phosphorus, and Iron Amendments on Water Extractable Lead and Arsenic in Orchard Soils. Communications in Soil Science and Plant Analysis, v.38, n.7, p.903-919. 2007.

Cunningham, S. D.; Lee, C. R. Phytoremediation: plant based remediation of contaminated soils and sediments. In: Skipper, H. D.; Turco, R. F. (ed.). Bioremediation: Science and applications. Madison: Soil Science Society of America, 1995. p.145-156 Special publication number 43.

Cunningham, S. D.; Own, D.W. Promises and prospects of phytoremediation. Plant Physiology, v.100, p.715-719, 1996. 
EPA - Environmental Protection Agency (United States). Acid digestion of sediments, sludges, and soils - Method 3050b, Washington: EPA, 1986. 12p.

Fayiga, A. O.; Ma, L.Q. Using phosphate rock to immobilize metals in soil and increase arsenic uptake by hyperaccumulator Pteris vittata. Science of The Total Environment, v.359, n.1 3, p.17-25. 2006.

Fayiga, A. O.; Ma, L. Q.; Zhou, Q. Effects of plant arsenic uptake and heavy metals on arsenic distribution in an arsenic contaminated soil. Air pollution and climate change: A Global Overview of the Effects on Forest Vegetation Environmental Pollution, v.147, n.3, p.737-742. 2007.

Goldberg, S.; Glaubig, R. A. Boron and silicon adsorption on an aluminum oxid. Soil Science American Journal, v.52, n.1, p.87-91, 1988.

Hering, J.; Chen, P.; Wilkie, M.; Elimelch, S.; Liang, S. Arsenic removal by ferric chloride. Journal American Water Works. v.88, n.4, p.155-167, 1996.

Jang, M.; Hwang, J. S.; Choi, S. I.; Park, J. K. Remediation of arsenic contaminated soils and washing effluents. Chemosphere, v.60, n.3, p.344-354. 2007.

Kumpiene, J.; Ore, S.; Renella, G.; Mench, M.; Lagerkvist, A.; Christian, M. Assessment of zerovalent iron for stabilization of chromium, copper, and arsenic in soil. Environmental Pollution, v.144, n.1, p.62-69, 2006.

Leist, M.; Casey, R. J.; Caridi, D. The management of arsenic wastes: problems and prospects. Journal of Hazardous Materials, v.76, p.125-138, 2000.

Mahimairaja S.; Bolan N. S.; Adriano D. C.; Robinson, B. Arsenic contamination and its risk management in complex environmental settings. Advances in Agronomy, v.86, p.82. 2005.

Masscheleyn, P. H.; Delaune, R. D.; Patrick, W. H. Jr. Effect of redox potential and $\mathrm{pH}$ on arsenic speciation and solubility in a contaminated soil. Environ. Science Technology, v.25, p.1414-1419, 1991.

Mesquita, A. A.; Amaral Sobrinho, N. M. B.; Oliveira, C.; Mazur, N.; Santos, S. F. Remediação de solos tratado com lodo rico em zinco. Revista Brasileira de Engenharia Agrícola e Ambiental, v.10, p.738-744, 2006.

Nguyen, T. V.; Vigneswaran, S.; Ngo, H. H.; Pokhrel, D.; Viraraghavan, T. Specific treatment technologies for removing arsenic from water. Engineering in Life Sciences, v.6, n.1, p.86-90. 2006.

Pinheiro, R. S. Inertização de resíduos perigosos através do uso de resíduos industriais alcalinos. Seropédica: UFRRJ, 2003. 68p. Dissertação Mestrado
Quazi, S.; Sarkar, D.; Khairom, A.; Datta, R. Arsenic bioavailability in a soil amended with water treatment residuals in the presence of phosphorus: Effect of amendment rates. In: The ASA CSSA SSSA International Annual Meetings, 2005. Salt Lake City: ASA CSSA SSSA, 2005. p.281

Sadiq, M. Arsenic chemistry in soils: na overview of thermodynamic predictions and field observations”. Water, Air and Soil Pollution, v.93, p.117-136, 1997.

Santos, F. S. dos; Amaral Sobrinho, N. M. B.; Mazur, N. Chemical amendment and phytoestabilization of industrial residue contaminated with $\mathrm{Zn}$ and Cd. Scientia Agricola, v.64, p.506-512, 2007.

Santos, F. S. dos; Hernandezallica, J. B. J. M.; Amaral Sobrinho, N. M. B., Mazur, N.; Garbisu, C. Chelate induced phytoextraction of metal polluted soils with brachiaria decumbens. Chemosphere, v.65, p.43-50, 2006.

Silva, W. G. P. da. Estudo da dinâmica química do arsênio no resíduo da companhia mercantil Ingá.. Seropédica: UFRRJ, 2005. 89p. Tese Doutorado

Spuller, C.; Weigand, H.; Marb, C. Trace metal stabilisation in a shooting range soil: Mobility and phytotoxicity. Stabilisation/ solidification treatment and remediation: Advances in S/S for waste and contaminated land. Journal of Hazardous Materials, v.141, n.2, p.378-387. 2007.

Tokunaga, S.; Wasay, S. A.; Park, S. Removal of arsenic (V) ion from aqueous solutions by lanthanum compounds. Water Science Technology, v.35, n.7, p.71-78,1997.

Wasay, S. A.; Parker, W.; Geel, P. J. van; Barrington, S.; Tokunaga, S. Arsenic pollution of a loam soil: retention form and decontamination. Journal of Soil Contamination, v.9, n.1, p.51-64, 2000.

Waychunas, G. A.; Rea, B. A.; Fuller, C. C.; Davis, J. A. Surface chemistry of ferrydrite: part I. EXAFS studies of the geometry of coprecipitated and absorbed arsenite. Geochimica et Cosmochimica Acta, v.57, p.2251-2269, 1993.

Wenzel, W. W.; Kirchbaumer, N.; Prohaska, B.; Stingeder, G.; Lombi, E.; Adriano, D.C. Arsenic fractionation in soil using an improved sequential extraction procedure. Analytica Chimica Acta, v.436, p.309-323, 2001.

Yang, L. Evaluation of in situ chemical fixation for remediation of arsenic contaminated soil. Tuscaloosa: University of Alabama, 2006, 151p. Ph.D. Dissertation

Yang, L.; Donahoe, R. J.; Redwine, J. C. In: situ chemical fixation of arsenic contaminated soils: An experimental study. Science of The Total Environment, v.387, n.1-3, p.28-41. 2007. 